\title{
On signature transition in Robertson-Walker cosmologies
}

\author{
K. Ghafoori-Tabrizi, S. S. Gousheh and H. R. Sepangi* \\ Department of Physics, Shahid Beheshti University, Evin, Tehran 19839, Iran
}

December 13, 2017

\begin{abstract}
We analyse a classical model of gravitation coupled to a self interacting scalar field. We show that, within the context of this model for Robertson-Walker cosmologies, there exist solutions in the spatially non-flat cases exhibiting transitions from a Euclidean to a Lorentzian spacetime. We then discuss the conditions under which these signature changing solutions to Einstein's field equations exist. In particular, we find that an upper bound for the cosmological constant exists and that close to the signature changing hypersurface, both the scale factor and the scalar field have to be constant. Moreover we find that the signature changing solutions do not exist when the scalar field is massless.
\end{abstract}

\section{Introduction}

The principle of causality is one of the most fundamental pillars on which classical as well as quantum physics is based. It exhibits itself in terms of the causal structure of a fourdimensional Lorentzian space-time manifold. Theories like quantum fields rely on this structure for a sensible interpretation of their results in a fixed background geometry. However, there is no apriori reason which would suggest that this causal structure should remain unaffected by the dynamical equations of general relativity. If we accept that in quantum cosmology [1], amplitudes for gravity can be expressed as sum of histories of 3-geometries [2] with different global 4-topological structures, then this may give rise to structures that cannot have pure Lorentzian or Euclidean geometries [3] and this implies a possible signature transition [4]. For example, an interesting feature of present day quantum cosmology [2] is characterised by its use of Riemannian signature spaces to arrive at an observable Lorentzian-signature spacetime.

*e-mail: hr-sepangi@cc.sbu.ac.ir 
Therefore, in order to gain further isight, it might be useful to study the dynamics of this phenomenon in simple models.

Traditionally, one of the features of classical gravity is that the signature of the metric is usually considered as fixed. This is not a property demanded by the field equations, but rather a condition one imposes on the metric before looking for solutions to Einstein's equations. If one relaxes this condition, one may find solutions to the field equations, which when interpreted suitably, exhibit a signature transition.

In general, for studying signature dynamics in the classical realm of gravity [5], one assumes that there exists a hypersurface which separates the space into two disjoint regions, one being Euclidean and the other Lorentzian. On this hypersurface, henceforth called the junction, the metric is either continuous and necessarily degenerate, or discontinuous with nondegenerate one-sided limits [6]. Therefore one can categorize this problem according to the continuity of the metric. Having chosen the form of the metric, there are two approaches for solving the field equations. The first (continuous) approach is to assume the validity of Einstein's field equations throughout and identify the form of the field equations at the junction with the junction conditions [7, 8, 9]. In the second (discontinuous) approach one finds solutions by solving Einstein's equations in disjoint regions next to the junction and then uses specific junction conditions (i.e. Darmois junction conditions) to match the solutions [10]. This furnishes an alternative way of categorizing the problem.

These different approaches have resulted in mainly two different sets of junction conditions which have led to a controversy between various authors [11]. Within the framework of Robertson-Walker cosmology using either approach, the scale factor, the scalar field and their first time derivatives are required to be continuous across the junction. However within the continuous approach, Hayward using a continuous metric has obtained extra conditions restricting the first time derivatives to be zero at the junction. The controversy mentioned above stems from the claims by some authors that these extra junction conditions are unnecessary, see e.g. 12, 13 and therefore the matter conservation need not hold under signature change [13].

Recently, Kossowski and Kriele [14], within the continuous approach, have proved a local existence and uniqueness theorem for solutions of Einstein's equations with dust energy momentum tensor in a class of m-dimensional signature changing spacetimes where initial conditions are given on the hypersurface of signature change. They have also proved a similar theorem in the case where the energy-momentum tensor represents a scalar field which is not self-interacting.

In this paper, we study a classical model where signature transition is possible, using the continuous approach with a continuous metric. This model is the same as that proposed by Dereli and Tucker [7] in which a real massive scalar field is taken as the matter source interacting with gravity and itself in a Robertson-Walker geometry whose signature evolution is controlled by a preferred coordinate. One then seeks solutions which are smooth and continuous across the junction on which the metric is degenerate. For the spatially flat universes, this results in exactly solvable Einstein's field equations [7]. Here we show that signature changing solutions also exist for arbitrary curvature in Robertson-Walker cosmologies. We do this by finding analytic solutions to the field equations close to the junction, and confirm and extend them away from the junction by a numerical method. We also show systematically the general 
set of restrictions that need to be imposed on the junction conditions and the parameters of this model including the cosmological constant. The general set of allowed conditions and parameters thus obtained include the ones used in reference [7] as a special case. In particular we find an upper bound on the cosmological constant, and the need for the scalar field to be massive in order to have continuous solutions. The present work is also complementary to [14] in that it solves the problem for a self-interacting scalar field. Having chosen a continuous approach, we obtain a set of junction conditions which, not surprisingly, are more restrictive than those obtained in the discontinuous approach. However, the restrictiveness of the junction conditions obtained here lies somewhere in between those obtained in [8] and [14]. We shall return to this point in the conclusions.

\section{$2 \quad$ Field equations}

We begin by briefly reviewing the model, and for comparison purposes, use the same notation as that of [7]. Consider gravity coupling to a scalar field through

$$
G=\kappa T[\phi]
$$

where the scalar field $\phi$ is a solution of

$$
\Delta \phi-\frac{\partial U}{\partial \phi}=0
$$

Here, $G=$ Ric $-\frac{1}{2} g \mathcal{R}$ is the Einstein tensor constructed from torsion-free connections compatible with the metric, and $U$ is a scalar potential for the real scalar field $\phi$ interacting with itself and gravity through the stress-energy tensor $T[\phi]$, given by

$$
T[\phi]=d \phi \otimes d \phi-\frac{1}{2} g(d \phi, d \phi) g-U(\phi) g .
$$

The above coupled equations are to be solved in a domain that would lead to Robertson-Walker cosmologies with Lorentzian signature. However, if the metric is suitably parametrized, one can expect to see continuous transition to a Euclidean domain. As in [7], we adopt a chart with coordinate functions $\left\{\beta, x^{1}, x^{2}, x^{3}\right\}$ where the hypersurface of signature change would be characterised by $\beta=0$. The metric can be parametrized to take the form

$$
g=-\beta d \beta \otimes d \beta+\frac{R^{2}(\beta)}{\left[1+(k / 4) r^{2}\right]^{2}} \sum_{i} d x^{i} \otimes d x^{i},
$$

where $r^{2}=\sum x_{i} x^{i}$, and depending on the values of $k=\{-1,0,1\}$ one would have an open, flat or closed universe, respectively. Now, it is apparent that the sign of $\beta$ determines the geometry, being Lorentzian if $\beta>0$ and Euclidean if $\beta<0$. For $\beta>0$, the traditional cosmic time can be recovered by the substitution $t=(2 / 3) \beta^{3 / 2}$. Adopting the chart $\left\{t, x^{i}\right\}$ and using equations (1) through (雨) with units in which $\kappa=1$, one finds

$$
3\left[\left(\frac{\dot{R}}{R}\right)^{2}+\frac{k}{R^{2}}\right]=\frac{\dot{\phi}^{2}}{2}+U(\phi),
$$




$$
\begin{aligned}
2\left(\frac{\dot{R}}{R}\right)^{\cdot}+3\left(\frac{\dot{R}}{R}\right)^{2}+\frac{k}{R^{2}} & =-\frac{\dot{\phi}^{2}}{2}+U(\phi), \\
\ddot{\phi}+3 \frac{\dot{R}}{R} \dot{\phi}+\frac{\partial U}{\partial \phi} & =0
\end{aligned}
$$

where a dot represents differentiation with respect to $t$. These equations are not all independent. Equation (6) can be obtained by combining equations (5) and (7). Equations (5) through (7) can also be derived in the context of Lagrangian dynamics. One defines the action 4 -form

$$
\Lambda=\left[\frac{1}{2} \mathcal{R}-\frac{1}{2} g(d \phi, d \phi)-U(\phi)\right] * 1,
$$

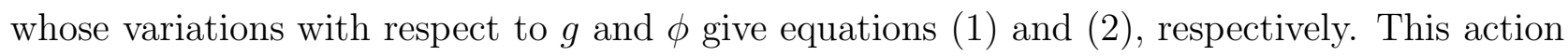
can be written as

$$
\Lambda=\mathcal{L} d t \wedge d x^{1} \wedge d x^{2} \wedge d x^{3}
$$

where

$$
\mathcal{L} d t=\left\{-3 R \dot{R}^{2}+3 k R+R^{3}\left[\dot{\phi}^{2} / 2-U(\phi)\right]\right\} d t+d\left(3 R^{2} \dot{R}\right) .
$$

After dropping the total derivative term and using the following transformation,

$$
\begin{gathered}
X=R^{3 / 2} \cosh (\alpha \phi), \\
Y=R^{3 / 2} \sinh (\alpha \phi),
\end{gathered}
$$

we obtain a mechanical analogue for this system given by the Lagrangian

$$
2 \alpha^{2} \mathcal{L} d t=\left\{-\dot{X}^{2}+\dot{Y}^{2}+\frac{9 k}{4}\left(X^{2}-Y^{2}\right)^{1 / 3}-2 \alpha^{2}\left(X^{2}-Y^{2}\right) U(\phi(X, Y))\right\} d t
$$

with $-\infty<\phi<\infty, 0 \leq R<\infty$ and $\alpha^{2}=\frac{3}{8}$. By a straightforward Legendre transformation we obtain the "Hamiltonian"

$$
2 \alpha^{2} \mathcal{H} d t=\left\{-\dot{X}^{2}+\dot{Y}^{2}-\frac{9 k}{4}\left(X^{2}-Y^{2}\right)^{1 / 3}+2 \alpha^{2}\left(X^{2}-Y^{2}\right) U(\phi(X, Y))\right\} d t,
$$

which we identify with the total energy density of the system. If we transform this expression back to the form of $R$ and $\phi$, we see that equation (5) is nothing more than a "zero energy condition." Of course any solution of the Euler-Lagrange equations would yield a constant total energy. However, Einstein's equations demand zero energy solutions only.

The above Lagrangian is considerably simplified if we take the potential to be

$$
2 \alpha^{2}\left(X^{2}-Y^{2}\right) U(\phi(X, Y))=a_{1} X^{2}+a_{2} Y^{2}+2 b X Y,
$$

where $a_{1}, a_{2}$ and $b$ are free parameters. This potential is required to have natural characteristics for small $\phi$, so that we may identify the coefficient of $\phi^{2} / 2$ in its Taylor expansion as a positive $m^{2}$ and $U(\phi=0)$ as a cosmological constant $\lambda$. In terms of the above parameters, $\lambda=\left.U\right|_{\phi=0}=$ 
$a_{1} /\left(2 \alpha^{2}\right)$ and $m^{2}=\partial^{2} U /\left.\partial \phi^{2}\right|_{\phi=0}=a_{1}+a_{2}$. The features of this potential have been discussed in more detail in [7].

The dynamical equations for $X$ and $Y$ in terms of the evolution variable $\beta$ now become

$$
\begin{aligned}
Y^{\prime \prime} & =\frac{1}{2}\left(\frac{1}{\beta}\right) Y^{\prime}-\frac{3}{4} \beta k Y\left(X^{2}-Y^{2}\right)^{-2 / 3}-\beta\left(a_{2} Y+b X\right), \\
X^{\prime \prime} & =\frac{1}{2}\left(\frac{1}{\beta}\right) X^{\prime}-\frac{3}{4} \beta k X\left(X^{2}-Y^{2}\right)^{-2 / 3}+\beta\left(a_{1} X+b Y\right),
\end{aligned}
$$

subject to the "zero energy condition"

$$
2 \alpha^{2} \mathcal{H}=\left(\frac{1}{\beta}\right)\left(-X^{\prime 2}+Y^{\prime 2}\right)-\frac{9}{4} k\left(X^{2}-Y^{2}\right)^{1 / 3}+\left(a_{1} X^{2}+a_{2} Y^{2}+2 b X Y\right)=0 .
$$

Here, a prime represents differentiation with respect to $\beta$. The coupled equations (16) and (17) must now be solved and, since their solutions render $\mathcal{H}$ a constant in $\beta$, equation (18) becomes only a restriction on the initial conditions. In the next section we first find analytic

solutions to these equations which are valid close to the junction, and then find the solutions for the full range of $\beta$ by a numerical method.

\section{Solutions}

\subsection{Analytic solutions close to the junction}

In this section we find analytic solutions which are valid near the junction, and obtain the restrictions imposed by equations (16)-(18) on the junction conditions. This is done by noting that in order to have well behaved solutions close to $\beta=0$, the first term of equation (18) shows that we must either have $X^{\prime}(\beta) \sim \beta^{n_{x}}$ and $Y^{\prime}(\beta) \sim \beta^{n_{y}}$, where $n_{x}, n_{y} \geq 1 / 2$, or $\left|X^{\prime}(0)\right|=\left|Y^{\prime}(0)\right|$. However, the first terms on the right hand side of equations (16) and (17) impose a more severe restriction. These two equations admit solutions $X^{\prime}(\beta) \sim \beta^{1 / 2}$ and $Y^{\prime}(\beta) \sim \beta^{1 / 2}$ close to $\beta=0$, however, these class of solutions do not admit real or $C^{2}$ solutions across $\beta=0$. One can show that regular solutions close to $\beta=0$ are of the form

$$
\begin{array}{lll}
X(\beta)=A_{x} \beta^{3}+X_{0} & \text { where } & A_{x}=\frac{2}{9}\left[-\frac{3}{4} \frac{k X_{0}}{\left(X_{0}^{2}-Y_{0}^{2}\right)^{2 / 3}}+a_{1} X_{0}+b Y_{0}\right] \\
Y(\beta)=A_{y} \beta^{3}+Y_{0} & \text { where } & A_{y}=\frac{2}{9}\left[-\frac{3}{4} \frac{k Y_{0}}{\left(X_{0}^{2}-Y_{0}^{2}\right)^{2 / 3}}-a_{2} Y_{0}-b X_{0}\right]
\end{array}
$$

with $X_{0} \equiv X(0)$ etc. Therefore, the initial conditions on the first and second derivatives must satisfy the relations

$$
X^{\prime}(0)=Y^{\prime}(0)=0 \quad \text { and } \quad X^{\prime \prime}(0)=Y^{\prime \prime}(0)=0 .
$$

Strictly speaking the conditions on the second derivatives are not initial conditions but rather consistency checks, since we have coupled second order equations. The above relations 
confine the possible solutions to a restricted class. Therefore, the initial values for the functions $X$ and $Y$ must now satisfy, c.f. equation (18)

$$
-\frac{9}{4} k\left(X_{0}^{2}-Y_{0}^{2}\right)^{1 / 3}+\left(a_{1} X_{0}^{2}+a_{2} Y_{0}^{2}+2 b X_{0} Y_{0}\right)=0 .
$$

Although equation (22) is equivalent to a sixth order algebraic equation which cannot be directly solved analytically, we can solve it by going back to the original variables $R$ and $\phi$. The solutions are either $R(0)=0$ giving $\phi(0)= \pm \infty$, which we exclude because we have been seeking continuous solutions across $\beta=0$, or $R(0) \neq 0$ (it is a free parameter) with

$$
\phi(0)=\frac{1}{2 \alpha} \cosh ^{-1}\left[\frac{D B \pm b \sqrt{D^{2}-B^{2}+b^{2}}}{B^{2}-b^{2}}\right],
$$

where

$$
D=\frac{9 k}{4 R(0)^{2}}-\frac{a_{1}-a_{2}}{2} \quad \text { and } \quad B=\frac{a_{1}+a_{2}}{2}=\frac{m^{2}}{2} .
$$

The contour plots of equation (22) for $k= \pm 1$ are given in figure 1. Along the contours, one finds the possible initial values for $X$ and $Y$. Of course, the acceptable values of $X$ and $Y$ ( $X>|Y|)$ can also be obtained analytically from equation (23).

In order for this model to have a valid quantum extension, at least in the scalar sector, we need to require the potential to have a minimum so that a real mass can be defined. That is

$$
\left.m_{s}^{2} \equiv \frac{\partial^{2} U}{\partial \phi^{2}}\right|_{\phi=\phi_{\min }}=m^{2} \sqrt{1-\frac{4 b^{2}}{m^{4}}} \quad \text { where } \quad \phi_{\min }=-\frac{1}{2 \alpha} \tanh ^{-1} \frac{2 b}{m^{2}} .
$$

Therefore we need to require $m^{2} \geq 2|b|$. This requirement along with the requirement of having real values of $X(0)$ and $Y(0)$ satisfying the zero energy condition and leading to real values for $R(0)(>0)$ and $\phi(0)$ give the following restriction on the parameters

$$
a_{1}-\frac{9 k}{4 R(0)^{2}} \leq|b| \leq \frac{a_{1}+a_{2}}{2} .
$$

It is important to note here that equations (19,20) imply

$$
\begin{aligned}
\dot{X}(0) & =0 \quad \text { and } \quad \dot{Y}(0)=0 \\
\ddot{X}(0) & =\frac{9}{2} A_{x} \text { and } \quad \ddot{Y}(0)=\frac{9}{2} A_{y} .
\end{aligned}
$$

These in turn lead to

$$
\begin{aligned}
& \dot{R}(0)=0 \quad \text { and } \dot{\phi}(0)=0, \\
& \ddot{R}(0)=\frac{k}{R(0)} \text { and } \quad \ddot{\phi}(0)=-\frac{m^{2}}{2 \alpha} \sinh [2 \alpha \phi(0)]-\frac{b}{\alpha} \cosh [2 \alpha \phi(0)] .
\end{aligned}
$$

The physical significance of these constraints and their relevance to the present controversy on the junction conditions will be discussed in the last section. 


\subsection{Numerical solution}

A noticeable feature of equations (16) and (17) is that for $k \neq 0$ they are singular at the critical values of $\beta\left(\beta_{c}\right)$ at which $Y\left(\beta_{c}\right)= \pm X\left(\beta_{c}\right)$. In terms of the original variables we have $R\left(\beta_{c}\right)=0$ and $\phi\left(\beta_{c}\right)= \pm \infty$. Any attempt in solving these equations involves handling these "moving singularities," as one encounters them when integrating the coupled equations. To handle these singularities, we establish jump conditions across them as follows: close to $\beta=\beta_{c}$, we assume that the solutions have the following linear forms

$$
\begin{aligned}
& X_{ \pm}=a_{ \pm}+b_{ \pm} \beta, \\
& Y_{ \pm}=c_{ \pm}+d_{ \pm} \beta,
\end{aligned}
$$

where \pm refers to the right or left hand sides of the singularity respectively. Substituting the above equations in (16) and (17) and integrating in the interval $\beta_{c}-\epsilon, \beta_{c}+\epsilon$, where $2 \epsilon$ is the distance across the jump, gives the following equation at $Y_{c}= \pm X_{c}$

$$
b_{+}-b_{-}=-\frac{9}{4} k \frac{\left(2 X_{c} \epsilon\right)^{1 / 3}}{\left(b_{-} \mp d_{-}\right)^{2 / 3}} \beta_{c}=\mp\left(d_{+}-d_{-}\right),
$$

where $\epsilon$ can be taken as small a value as is desired for any required accuracy. Moreover, the values of $a_{ \pm}$and $c_{ \pm}$are determined by the requirement of the continuity of $X$ and $Y$ at $\beta_{c}$. Equation (29) establishes our jump condition for handling the singularities of the differential equations. For ease of comparison with the solutions of the spatially flat $(k=0)$ RobertsonWalker universe, we use the same set of parameters as in [7] $\left(b=2, \lambda=0, m^{2}=4.5\right)$ and choose our initial conditions according to equations (21) and (22). We note that since equation (18) is a constant of motion, if it is satisfied at $\beta=0$, it will be satisfied at all other values of $\beta$. For integrating equations (16) and (17), we have used the fourth order Runge-Kutta method. The resulting solutions for $k= \pm 1$ are shown in figure 2. As a measure of the accuracy of the solutions, we have computed the "zero energy condition," equation (18), as a function of $\beta$ for $k= \pm 1$. We have found that the values of "total energy" stay very close to zero in the full range of $\beta$ shown in figure 2 , thus indicating the validity of our numerical solutions. As a further check, we have numerically recovered the analytic solutions presented in [7] for $k=0$. In figure 3 the variations of $\phi$ and $R$ and in figure 4 that of the curvature scalar are shown as a function of $\beta$.

\section{Conclusions}

We have shown that in the context of classical general relativity, using a model proposed in [7], there could exist signature transitions in Robertson-Walker cosmologies with arbitrary curvature. In this model, these transitions are embodied in the form of smooth continuous functions describing solutions to Einstein field equations coupled to a scalar field. These solutions describe a geometry in which the covariant metric tensor is degenerate on a hypersurface characterised by $\beta=0$ and undergoes a transition from a Euclidean to a Lorentzian signature.

\footnotetext{
${ }^{1}$ None of the values of $\beta_{c}$ coincide with the junction at which $\beta=0$.
} 
As in the $k=0$ case, the singular behaviour of the scalar field characterizes the beginning of the Euclidean domain. This feature is also reflected in the behaviour of the curvature scalar. For $k=0$ the solutions for $X$ and $Y$ are not singular anywhere in the Lorentzian domain. However, for $k \neq 0$, there are singularities at $Y= \pm X$ but they are very mild and show up only at the second derivative level. As $k$ increases we observe more rapid oscillations in $X$ and $Y$, leading to more rapid variations in all the physical quantities. However, for all values of $k$, they vary extremely slowly in the vicinity of the signature changing hypersurface, as can be seen from figures 3 and 4.

As far as the controversy on the junction conditions in the current literature is concerned, we find that in our continuous approach to signature change (with a continuous metric) the restrictions on the initial conditions are given by equations (21,27,28). These conditions are slightly more restrictive than those of [8], because equation (21) implies that the second derivatives of $R$ and $\phi$ with respect to the evolution parameter $(\beta)$ are zero, whereas the analysis presented there does not seem to require this. However, the junction conditions of [14] seem to be too restrictive since it requires the second (cosmic) time derivatives of $R$ and $\phi$ to be zero, contrary to equation (28). As was mentioned before, we only require the second derivatives with respect to $\beta$ to be zero.

We now discuss the physical significance of the restrictions on the parameters of the model which is presented in equation (24). When this equation is written in terms of the physical parameters it becomes

$$
\frac{3 \lambda}{4}-\frac{9 k}{4 R^{2}(0)} \leq|b| \leq \frac{m^{2}}{2} .
$$

In particular we have $\lambda_{\max }=3 k / R^{2}(0)+2 m^{2} / 3$ and this implies that $\lambda$ cannot have an arbitrarily large positive value. Rather more importantly, note that as $m \rightarrow 0$, equation (30) implies that $b=0$ and subsequently equation (23) shows that $\phi(0) \rightarrow \pm \infty$. Therefore, in order to have continuous solutions across the junction, the scalar field has to be massive. We expect this result to be independent of the particular choice of the potentual $U(\phi)$.

It is interesting to note that although the solutions for $k=0$ and $k= \pm 1$ are qualitatively not very different, there is a pronounced difference in the curvature scalar. That is $\mathcal{R}(0)=$ $6 k / R^{2}(0)$.

\section{Acknowledgements}

We would like to thank R. W. Tucker for motivating us to do this problem and F. Ebrahimi for useful discussions.

\section{References}

[1] B. S. DeWitt, Phys. Rev. 160, 1113 (1967); C. W. Misner, Phys. Rev. 186, 1319(1969); W. F. Blyth and C. J. Isham, Phys. Rev. D 11, 768 (1975).

[2] J. B. Hartle and S. W. Hawking, Phys. Rev. D 28, 2960 (1983); S. W. Hawking, Nucl. Phys. B239, 257 (1984); S. W. Hawking, 300 years of gravitation ed. S. W. Hawking and 
W. Israel, Cambridge University Press 631 (1987); J. J. Halliwell and J. B. Hartle, Phys. Rev. D 41, 1815 (1990); G. W. Gibbons and J. B. Hartle, Phys. Rev. D 42, 2458 (1990); R. Kerner and J. Martin, Class. Quantum Grav. 10, 2111 (1993); J. Martin, Phys. Rev. D 49, 5086 (1994).

[3] W. Kundt, Commun. Math. Phys. 4, 143 (1966); R. P. Geroch, J. Math. Phys. 8, 782 (1967); D. Brill, Magic without magic, ed. J. K. Louder (San Fransisco: Freeman) 309 (1972).

[4] S. W. Hawking and I. G. Moss, Phys. Lett. 110B, 35 (1982); A. Vilenkin, Phys. lett. 117B, 25 (1982); A. Vilenkin, Phys. Rev. D 27, 2848 (1983); A. D. Linde, Phys. Lett. 162B, 281 (1985); A. Vilenkin, Phys. Rev. D 33, 3560 (1986); I. Moss and S. Poletti, Nucl. Phys. B341, 155 (1990).

[5] A. D. Sakharov, Sov. Phys.-JETP 60, 214 (1984); G. F. R. Ellis, A. Sumruk, D. Coule and C. Hellaby, Class. Quantum Grav. 9, 1535 (1992).

[6] M. Kossowski and M. Kriele, Class. Quantum Grav. 10, 2363 (1993); T. Dray, J. Math. Phys. 37, 5267 (1996).

[7] T. Dereli and R. W. Tucker, Class. Quantum Grav. 10, 365 (1993).

[8] S. A. Hayward, Class. Quantum Grav. 9, 1851 (1992); ibid 2453(E); S. A. Hayward, Class. Quantum Grav. 10, L7 (1993).

[9] F. Darabi and H. R. Sepangi, Class. Quantum Grav. 16, 1565 (1999).

[10] C. Hellaby and T. Dray, Phys. Rev. D 49, 5096 (1994); T. Dray and C. Hellaby, J. Math. Phys. 35, 5922 (1994).

[11] S. A. Hayward, phys. Rev. D 52, 7331 (1995); C. Hellaby and T. Dray, phys. Rev. D 52, 7333 (1995).

[12] G. F. R. Ellis, Gen. Rel. Grav. 24, 1047 (1992).

[13] T. Dray, C. A. Manogue, and R. W. Tucker, Gen. Rel. Grav. 23, 967 (1991); T. Dray, C. A. Manogue and R. W. Tucker, Phys. Rev. D 48, 2587 (1993); C. Hellaby and T. Dray, Phys. Rev. D 49, 5096 (1994).

[14] M. Kossowski and M. Kriele Proc. R. Soc. Lond. A 446, 115 (1995). 


\section{Figure Captions}

Figure 1: The contour plots of the allowed values of $X$ and $Y$, satisfying the equation of constraint (22) at $\beta=0$ for $k= \pm 1$ - figures $1(\mathrm{a}), 1(\mathrm{~b})$. The point $(0,0)$ is a solution and the curves approaching this point actually pass through it, although this is not shown on the plots due to the limitations on the numerical accuracy.

Figure 2: Solutions for $X$ (broken curve) and $Y$ (solid curve) as a function of $\beta$, for $k= \pm 1$ - figures 2(a), 2(b). The values of the parameters are $b=2, \lambda=0, m^{2}=4.5$.

Figure 3: Variation of $\phi$ and $R$ with $\beta$ for $b=2, \lambda=0, m^{2}=4.5$, and $k=1$ (broken curves) and $k=-1$ (solid curves). Note that $\phi$ and $R$ vary extremely slowly in the vicinity of $\beta=0$.

Figure 4: Variation of the scalar curvature $\mathcal{R}$ with $\beta$ for $b=2, \lambda=0, m^{2}=4.5$, and $k=1$ (broken curve) and $k=-1$ (solid curve). Note that $\mathcal{R}(0)=6 k /\left[R^{2}(0)\right]$. 


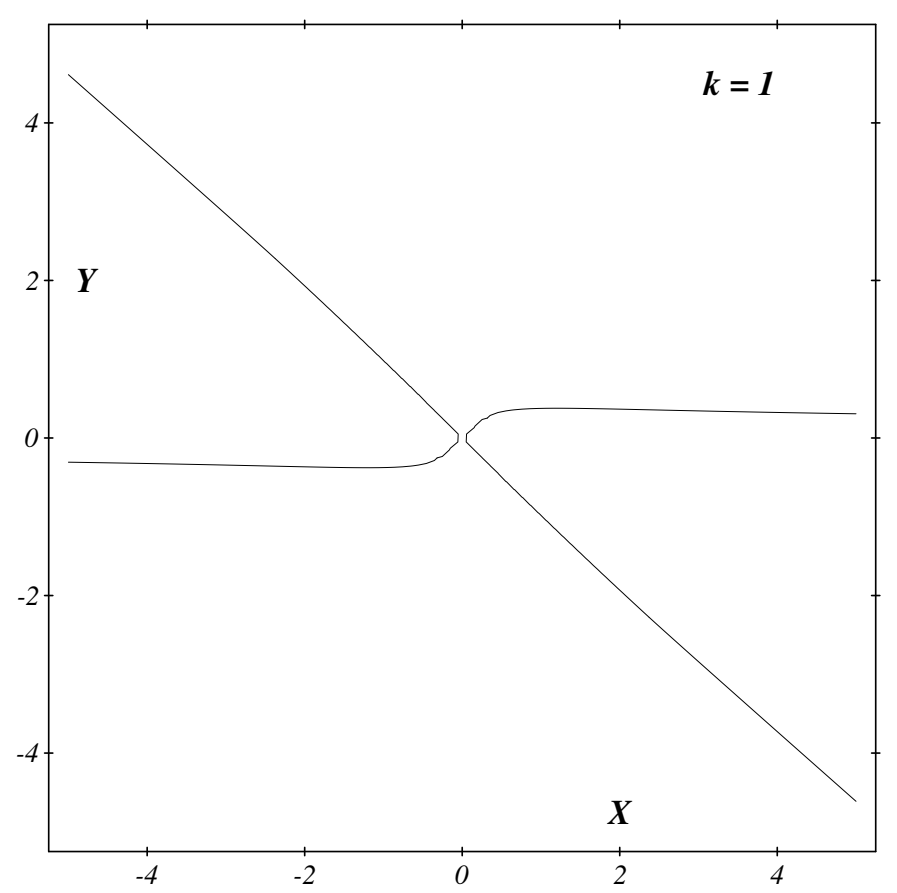

(a)

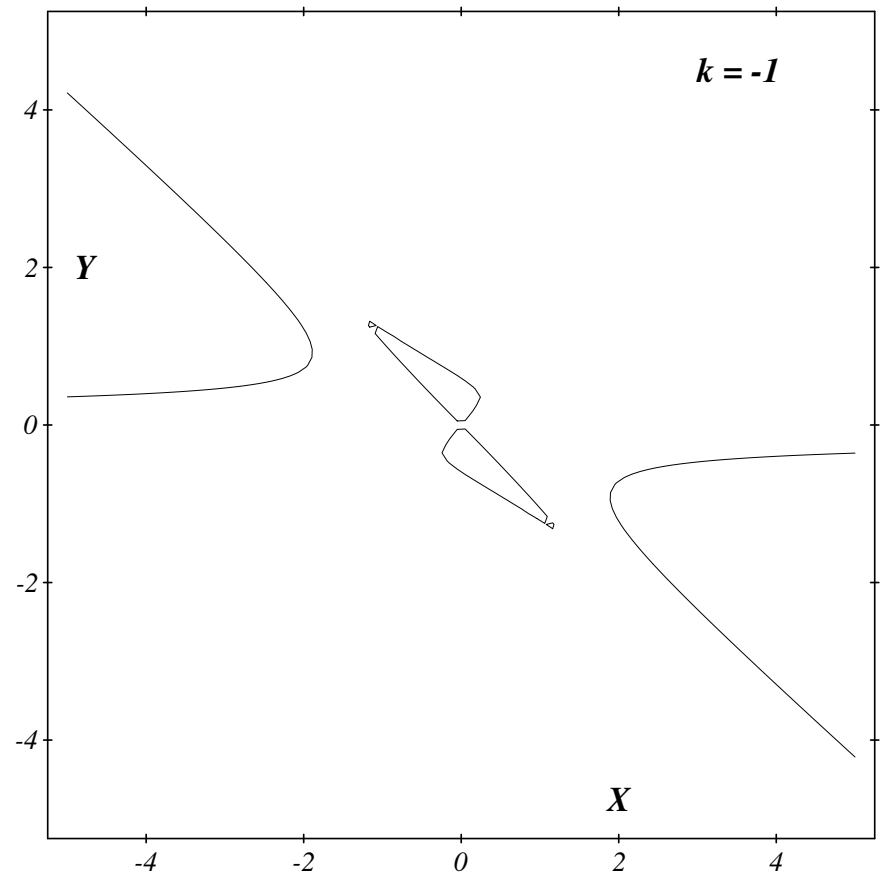


Figure 2 .

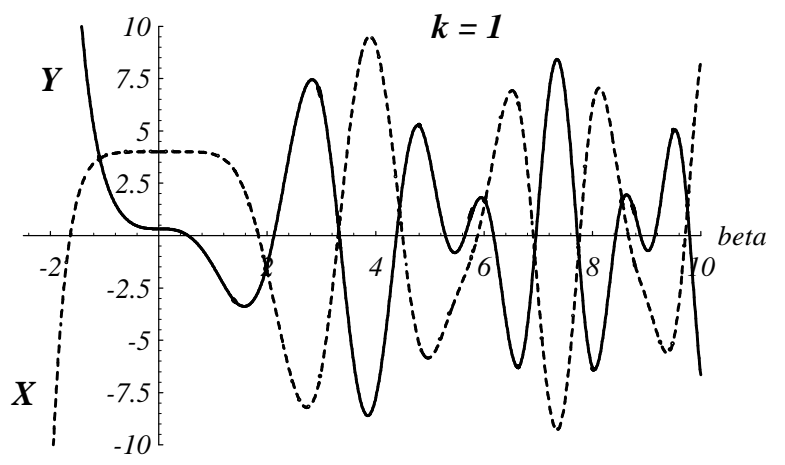

(a)

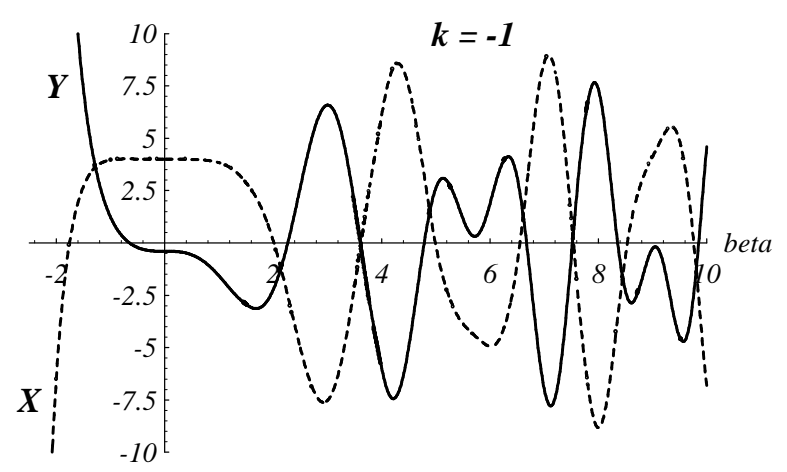

(b) 
Figure 3.

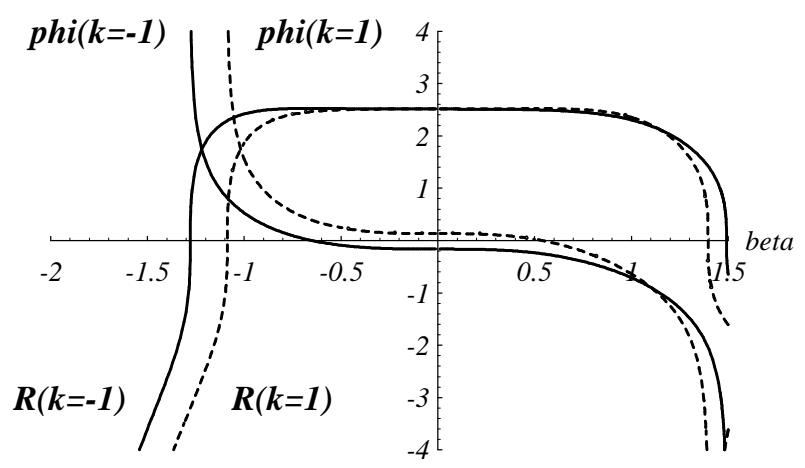


Figure 4.

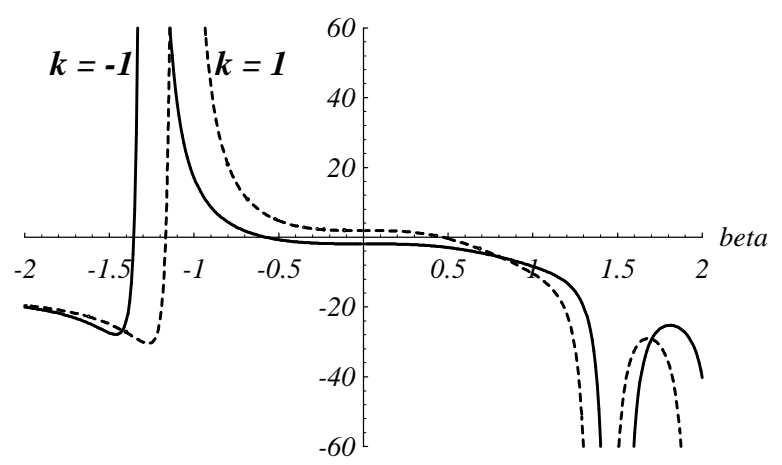

